Bundesgesundheitsbl - Gesundheitsforsch Gesundheitsschutz 2009 · 52:257 DOI 10.1007/s00103-009-0784-x Online publiziert: 12. Februar 2009 (c) Springer Medizin Verlag 2009

\title{
Einstufung Wasser gefährdender Stoffe
}

ihren Sitzungen 2008 für folgende Stoffe Wassergefährdungsklassen (WGK) empfohlen. Diese Bewertungen werden dem BMU zur Bekanntmachung in der nächs- ten Fortschreibung der Verwaltungsvorschrift Wasser gefährdender Stoffe (VwVwS) vorgeschlagen. 促 Umwelt, Natursch und Reaktorsicherheit (BMU) hat auf

Umstufungen in Anhang 1 oder Anhang 2 der VwVwS vom 27.7.2005 vom 27.7.2005

\begin{tabular}{|llll|}
$\begin{array}{l}\text { CAS- } \\
\text { Nummer }\end{array}$ & Stoffbezeichnung & $\begin{array}{l}\text { Kenn- } \\
\text { Nummer }\end{array}$ & WGK \\
\hline $556-67-2$ & Octamethylcyclotetrasiloxan & 7735 & 3 \\
\hline $7027-11-4$ & $\begin{array}{l}\text { Cyclohexancarbonitril, } \\
1,3,3-t r i m e t h y l-5-0 x 0-\end{array}$ & 4478 & 3 \\
\hline $\begin{array}{l}\text { Methacrylsäureester C12-C22, } \\
142-90-5\end{array}$ & 7779 & 1 \\
$32360-05-05-7$ & gesättigt, linear und verzweigt & & \\
$45294-18-6$ & & & \\
$85736-97-6$ & & & \\
$90551-84-1$ & & & \\
$90552-04-8$ & & & 1 \\
\hline $32804-77-6$ & 2-Ethoxyethylcyanacetat & 7754 & 1 \\
\hline
\end{tabular}

\begin{tabular}{|llll|}
\hline CAS-Nummer & Stoffbezeichnung & Kenn-Nummer & WGK \\
\hline $7719-12-2$ & Phosphortrichlorid & 1245 & 1 \\
\hline
\end{tabular}

Rückfragen sind zu richten an: Geschäftsstelle der Kommission Bewertung Wasser gefährdender Stoffe (KBwS) im Umweltbundesamt, Schichauweg 58, 12307 Berlin,

Internetpräsenz: http//www.umweltbundesamt.de/wgk.htm, E-Mail-Adresse: wgk@uba.de 